\begin{tabular}{|c|c|c|c|}
\hline \multicolumn{4}{|c|}{ Table 1: Characteristics of the patients } \\
\hline Age - years & $69 \pm 12$ & $\mathrm{CHA}_{2} \mathrm{DS}_{2}$-VASC score & number of patients \\
\hline Male gender - no (\%) & $78(62 \%)$ & 0 & $23(18 \%)$ \\
\hline Hypertension & $82(65 \%)$ & 1 & $20(16 \%)$ \\
\hline Diabetes Mellitus & $15(12 \%)$ & 2 & $32(25 \%)$ \\
\hline Coronary disease & $12(10 \%)$ & 3 & $24(19 \%)$ \\
\hline Heart failure & $2(2 \%)$ & 4 & $16(13 \%)$ \\
\hline $\mathrm{BMI}>30$ & $54(43 \%)$ & 5 & $7(6 \%)$ \\
\hline Excess alcohol & $46(37 \%)$ & 6 & $3(2 \%)$ \\
\hline OSA & $28(22 \%)$ & 7 & $1(1 \%)$ \\
\hline
\end{tabular}

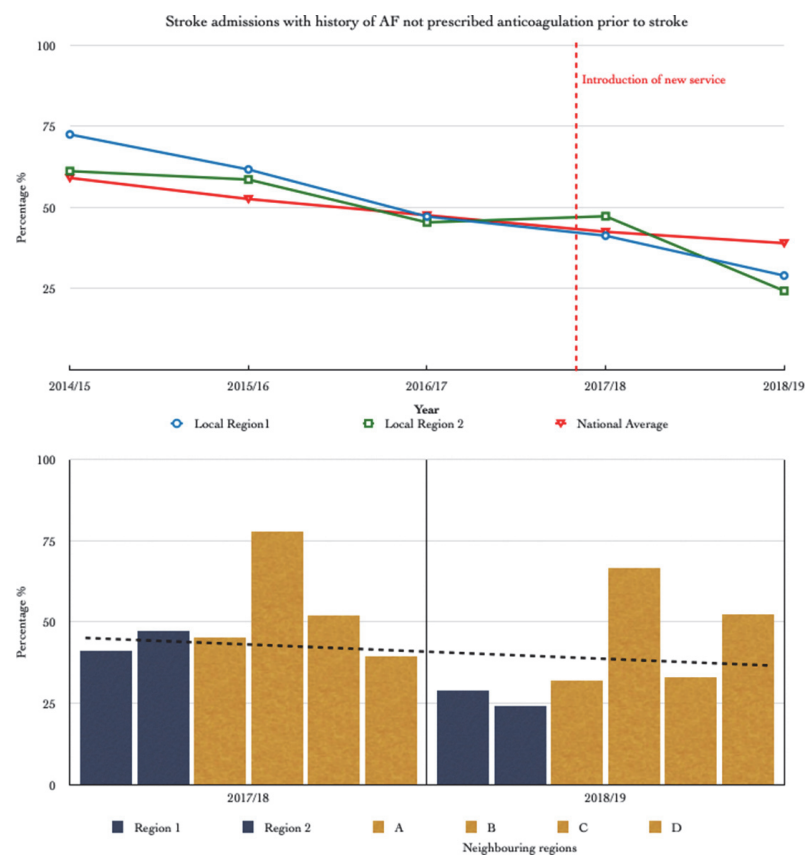

Abstract 84 Figure 1

with no major contra-indications) received oral anticoagulation. Over 12 months follow-up, rates of TIA/stroke was low $(n=3$, 2.4\%). All-cause mortality was also low $(n=1,0.8 \%)$. NHS England region-wide data showed a marked reduction in percentage of total stroke admissions with history of AF not taking anticoagulation at stroke presentation in 2018/19 as compared to $2017 / 18$ across our two regions; this was lower than the national average and the lowest within West Yorkshire (figure 1$)^{2}$

Conclusions An integrated service for AF management implemented community-wide was associated with high uptake of oral anticoagulation among eligible patients and low rates of AF-related hospitalisations and all-cause mortality. Across the community, among stroke admissions with AF at presentation, a lower percentage of patients without anticoagulation was observed in comparison with previous years and neighbouring regions.

Conflict of Interest none

\section{PROGNOSTIC VALUE OF PREMATURE VENTRICULAR COMPLEXES DURING EXERCISE STRESS TESTING IN 56,000 INDIVIDUALS WITHOUT KNOWN STRUCTURAL HEART DISEASE FROM UK BIOBANK: IS A SINGLE ECTOPIC PROGNOSTIC?}

${ }^{1}$ Stefan van Duijvenboden, ${ }^{2} J u l i a$ Ramirez, ${ }^{1}$ Michele Orini, ${ }^{2}$ Andrew Tinker, ${ }^{2}$ Patricia Munroe, ${ }^{1}$ Pier Lambiase. ' University College London, London, UK; ${ }^{3}$ Queen Mary University of London

\subsection{6/heartjnl-2021-BCS.85}

Introduction Premature ventricular complexes (PVCs) are frequently detected on routine electrocardiogram monitoring during exercise. The prognostic implications of frequent or repetitive PVCs during exercise are well recognised in individuals with known heart disease, but conflicting results have been reported in individuals without known structural or ischaemic substrates. The aim of this study was to evaluate the relationship between PVC frequency during exercise and the risk of major adverse cardiovascular events (MACE) in a large cohort of $\sim 56,000$ individuals without known cardiovascular disease with long-term follow-up.

Methods Approximately 77,000 individuals from the UK Biobank study participated in an exercise test using a stationary bicycle in conjunction with a 4-lead electrocardiograph device. We excluded individuals with a known cardiovascular disease or a corrupt/incomplete ECG registration. The number of PVCs in the ECG registration were counted for the remaining individuals $(\mathrm{N}=56,176)$ using an in-house developed deep learning algorithm. All registrations with a PVC frequency of $\square 5 \%$ were reviewed manually and corrected if needed. The study endpoint was MACE, defined as either hospitalization or death due to myocardial infarction, heart failure, or ventricular arrhythmia. The association with MACE was evaluated for a range of PVC counts (up to 30 PVCs during exercise) using uni- and multivariate Cox regression analysis. In the multivariate model we adjusted for established cardiovascular risk factors, including age, sex, body mass index, smoking, diabetes, and heart rate recovery.

Results The mean age of all individuals was 56,7 ( \pm 8.2) years and men and women were relatively even numbered (47\% men). The median follow-up was 10 years during which $\mathrm{N}=1,871$ (3.3\%) individuals reached the study endpoint. The prevalence of PVCs observed during the stress test ranged from $\mathrm{N}=16,330 \quad(29 \%)$ individuals having $\square 1 \mathrm{PVC}$, to $\mathrm{N}=1758 \quad(\mathrm{x} \%)$ and $\mathrm{N}=856 \quad(1.5 \%)$ for $>10 \%$ and $\square 25 \%$ PVCs, respectively. Interestingly, having at least 1 PVC was already significantly associated with adverse outcome: hazard ratio (HR) [95\% CI]: $1.6[1.4-1.7], \mathrm{p}<0.001$, (unadjusted), and $1.3[1.2-1.4], \mathrm{p}<0.001$ (adjusted) with respect to individuals showing no PVCs. We observed a clear dose response relationship between the PVC count and associated risk as shown in Figure 1, with the highest measured risk for the highest tested PVC count of $\square 30$ : HR [95\% CI]: 3.1 [2.4 - 4.0], p $<0.001$, (unadjusted), 1.9 [1.5 - 2.5], $\mathrm{p}<0.001$ (adjusted).

Conclusion Historically, occasional occurrence of PVCs during exercise has been considered benign. However, the results of this study may suggest that also low PVC frequencies may carry relevant prognostic information for cardiovascular disease independent of other cardiovascular risk factors. The mechanisms remain to be investigated, but may include subclinical changes in myocardial functioning.

Conflict of Interest None 